\title{
Two-dimensional modulation instability of wind waves
}

\author{
Roger Grimshaw ${ }^{1}$ (D)
}

Received: 4 July 2019 / Accepted: 16 September 2019 / Published online: 25 September 2019

(c) The Author(s) 2019

\begin{abstract}
It is widely known that deep-water waves are modulationally unstable and that this can be modelled by a nonlinear Schrödinger equation. In this paper, we extend the previous studies of the effect of wind forcing on this instability to water waves in finite depth and in two horizontal space dimensions. The principal finding is that the instability is enhanced and becomes super-exponential and that the domain of instability in the modulation wavenumber space is enlarged. Since the outcome of modulation instability is expected to be the generation of rogue waves, represented within the framework of the nonlinear Schrödinger equation as a Peregrine breather, we also examine the effect of wind forcing on a Peregrine breather. We find that the breather amplitude will grow at twice the rate of a linear instability.
\end{abstract}

\section{Introduction}

It is now widely known that water waves in deep water are unstable to long-wave perturbations leading to modulation instability, which can lead to the formation of rogue waves. Often, this process is modelled by the nonlinear Schrödinger equation, and then, rogue waves may represented by Peregrine breathers, see, for instance, Kharif et al. (2009), Osborne (2010), Grimshaw and Tovbis (2013). In one horizontal space dimension, water wave packets are represented by

$$
\begin{aligned}
& \zeta=\epsilon A(X, T) \exp (i \theta)+\text { c.c. }+\cdots, \\
& \text { where } \theta=k x-\omega(k) t, X=\epsilon\left(x-c_{g} t\right), T=\epsilon^{2} t . \\
& \omega^{2}(k)=\frac{g}{h} q \sigma, \quad c_{g}=\omega_{k}=\frac{\omega}{2 k}\left\{1+\frac{q}{\sigma}\left(1-\sigma^{2}\right)\right\}, \\
& \quad q=k h, \quad \sigma=\tanh q .
\end{aligned}
$$

Here, $\zeta(x, t)$ is the surface elevation, and $\epsilon \ll 1$ is a small parameter measuring the wave amplitude. An asymptotic expansion in which the linear dispersive effects are scaled to balance the leading order nonlinear effects leads to the nonlinear Schrödinger equation, see Hasimoto and Ono (1972):

$$
i A_{T}+\delta A_{X X}+\mu|A|^{2} A=0, \quad \delta=\frac{c_{g k}}{2} .
$$

Roger Grimshaw

r.grimshaw@ucl.ac.uk

1 Department of Mathematics, University College London, London, UK
The coefficient $\mu$ of the nonlinear term is given by

$$
\begin{aligned}
\mu= & -\frac{k^{2} \omega}{4 \sigma^{4}}\left(9 \sigma^{4}-10 \sigma^{2}+9\right) \\
& +\frac{\omega^{3}}{2 \sigma^{3}\left(g h-c_{g}^{2}\right)}\left(2 \sigma\left(3-\sigma^{2}\right)\right. \\
& \left.+3 q\left(1-\sigma^{2}\right)^{2}\right) .
\end{aligned}
$$

Note that the first term in (5) is always negative and the second term in (5) is always positive. In deep water $(q \rightarrow \infty)$, the second term vanishes, and the coefficient $\mu \rightarrow-2 \omega k^{2}<0$. In general, $\mu<0(>0)$ according as $q>q_{c}\left(q<q_{c}\right)$, where $q_{c}=1.363$, and with equality between the two terms occurring at $q=q_{c}$. There is modulation instability when $\mu \delta>0$, that is, since here $\delta<0$ when $\mu<0$ and so $q>q_{c}$. The leading order omitted terms in (1) are the $O\left(\epsilon^{2}\right)$ second harmonic and mean flow terms.

Our concern in this paper is with the effect of wind forcing on modulation instability. Then, the amplitude $A$ satisfies a forced nonlinear Schrödinger equation, see, for instance, Leblanc (2007), Touboul et al. (2008), Montalvo et al. (2013), Brunetti et al. (2014), Slunyaev et al. (2015), Grimshaw (2018, 2019):

$i A_{T}+\delta A_{X X}+\mu|A|^{2} A=i \Delta A$,

Wind forcing is represented by the addition to (4) of a linear growth rate term. The coefficient $\Delta$ depends on the wind shear profile inter alia, and various expressions can be found in the literature, the most well known being that originally 
derived by Miles (1957) and subsequently adapted and modified in various ways, see, for instance, Miles (1993), Morland and Saffman (1993), Janssen (2004), Stiassnie et al. (2007), Sajjadi et al. (2014), Zakharov et al. (2017), Grimshaw (2018). Our concern here is not with the precise form of $\Delta$, but the effect of this term on modulation instability. This has been examined by Leblanc (2007), Touboul et al. (2008), Montalvo et al. (2013), Brunetti et al. (2014), Slunyaev et al. (2015), and Grimshaw (2018) in the one space-dimension setting for deep-water waves, and recently by Grimshaw (2019) for deep-water waves in two-space dimensions.

In this paper that is extended to water waves in finite depth and in two-space dimensions. In Sect. 2, we present the forced Benney-Roskes system for the description of nonlinear water wave packets forced by wind in two horizontal space dimensions. In Sect. 3.1, we analyse the modulation instability, and in Sect. 3.2, we examine the effect of wind forcing on a Peregrine breather. We conclude in Sect. 4.

\section{Forced Benney-Roskes equation}

When the effects of modulation in the transverse $y$-direction are taken into account, the wave amplitude is given by $A(X, Y, T)$, where $Y=\epsilon y$. The forced nonlinear Schrödinger equation (6) is then replaced by a forced Benney-Roskes system:

$i A_{T}+\delta A_{X X}+\delta_{1} A_{Y Y}+\mu|A|^{2} A+Q A=i \Delta A$,

$\alpha Q_{X X}+Q_{Y Y}+\beta\left(|A|^{2}\right)_{Y Y}=0$,

where $\delta_{1}=\frac{c_{g}}{2 k}, \quad \alpha=1-\frac{c_{g}^{2}}{g h}$.

Here, $Q$ is an induced mean flow term. The coefficients $\mu, \delta, \Delta$ are those defined in (6) and the coefficient $\beta$ is given by

$$
\left(g h-c_{g}^{2}\right) \beta=-\frac{2 \omega^{3}}{\sigma^{2}}\left(1+\frac{c_{g}}{2 c}\left(1-\sigma^{2}\right)\right)^{2} .
$$

In the deep-water limit $q \rightarrow \infty$. $\beta \rightarrow 0 . Q \rightarrow 0$ and the system $(7,8)$ reduces to the forced two-dimensional nonlinear Schrödimger equation studied by Grimshaw (2019). In the absence of forcing, this system $(7,8)$ was derived by Benney and Roskes (1969), see also Davey and Stewartson (1974), Djordjevic and Redekopp (1977), and the review by Grimshaw (2007). Here, we have added a forcing term by analogy with the forced nonlinear Schrödimger equation (6). The system $(7,8)$ is not integrable in general, but can be shown to reduce to an integrable Davey-Stewartson system in the shallow-water limit $q \rightarrow 0$ and in the absence of the forcing term. The coefficients $\delta<0, \delta_{1}>0, \beta<0$ and $\alpha>0$, so that the equation for $A$ is "hyperbolic", but that for
$Q$ is "elliptic". In addition, when we recall that $\mu<0(>0)$ according as $q>q_{c}\left(<q_{c}\right), q_{c}=1.363$ we see that the equation for $A$ is focussing for " $x$ " and defocussing for " $y$ " when $q>q_{c}$, but is defocussing for " $x$ " and focussing for " $y$ " when $q<q_{c}$.

The nonlinear and dispersive terms in (6) are not sufficient to control the exponential growth of a localised wave packet, since

$\frac{\mathrm{d}}{\mathrm{d} T} \int_{-\infty}^{\infty} \int_{-\infty}^{\infty}|A|^{2} \mathrm{~d} X \mathrm{~d} Y=2 \Delta \int_{-\infty}^{\infty} \int_{-\infty}^{\infty}|A|^{2} \mathrm{~d} X \mathrm{~d} Y$.

Furthermore, the modulation instability, present when $\mu \delta>$ 0 (as for deep-water waves) in the absence of wind, is enhanced in the presence of wind, see Leblanc (2007), Touboul et al. (2008), Montalvo et al. (2013), Brunetti et al. (2014), Slunyaev et al. (2015), Grimshaw (2018) for the one-dimensional case and 'Grimshaw (2019) for the two-dimensional case in deep water. Here, we extend that analysis to the two-dimensional case in finite depth using the Benney-Roskes system $(7,8)$. By analogy with the analysis of Grimshaw (2019), we make the transformations:

$$
\begin{aligned}
A & =B \exp (\Delta T), \quad Q=R \exp (2 \Delta T), \\
s & =\frac{\exp (2 \Delta T)-1}{2 \Delta},
\end{aligned}
$$

Then, the system $(7,8)$ becomes

$$
\begin{aligned}
& i B_{S}+\delta F B_{X X}+\delta_{1} F B_{Y Y}+\mu|B|^{2} B+R B=0, \\
& \alpha R_{X X}+R_{Y Y}+\beta\left(|B|^{2}\right)_{Y Y}=0, \\
& \text { where } F=\frac{1}{1+2 \Delta s}=\exp (-2 \Delta T) .
\end{aligned}
$$

In this transformed system, the energy expression (11) becomes a conservation law:

$\frac{\mathrm{d}}{\mathrm{d} T} \int_{-\infty}^{\infty} \int_{-\infty}^{\infty}|B|^{2} \mathrm{~d} X \mathrm{~d} Y=0$

\section{Modulation instability}

\subsection{Linearised analysis}

The transformed system $(13,14)$ has the "plane wave" solution:

$B=B_{0} \exp \left(i \mu\left|B_{0}\right|^{2} s\right), \quad R=0$.

In the absence of wind forcing, this is just the usual Stokes wave. Modulation instability is then found by putting $B=$ 
$B_{0}(1+b) \exp \left(i \mu\left|B_{0}\right|^{2} s\right)$ into $(13,14)$ and linearizing in $b, R$, so that

$$
\begin{aligned}
& i b_{s}+\lambda F b_{X X}+\sigma F b_{Y Y}+\mu\left|B_{0}\right|^{2}\left(b+b^{*}\right)+R=0 . \\
& \alpha R_{X X}+R_{Y Y}+\beta\left|B_{0}\right|^{2}\left(b+b^{*}\right)_{Y Y}=0 .
\end{aligned}
$$

Then, we put $b=(p(s)+i q(s)) \cos (K X+L Y)$, where $p, q$ are real-valued, and likewise $R=r(s) \cos (K X+L Y)$, so that

$$
\begin{aligned}
& p_{s}-M F q=0, \\
& q_{s}+M F p-2 \mu\left|B_{0}\right|^{2} p+r=0 . \\
& N r+2 \beta L^{2}\left|B_{0}\right|^{2} p=0 . \\
& M=\delta K^{2}+\delta_{1} L^{2}, \quad N=\alpha K^{2}+L^{2} .
\end{aligned}
$$

Eliminating $q, r$ yields

$$
\left\{\frac{p_{s}}{F}\right\}_{s}+\left(M F-2 v\left|B_{0}\right|^{2}\right) M p=0, \quad v=\mu+\frac{\beta L^{2}}{N}
$$

When there is no wind forcing, so that $\Delta=0$, then $F=$ 1 and this yields the well-known criterion for modulation instability, namely, that $M\left(M-2 v\left|B_{0}\right|^{2}\right)<0$. If further $L=0, v=\mu$ and this yields modulation instability for $\mu \delta>0$. Since here, $\delta<0$, there is instability only for $\mu<0$, that is for deep-water waves $q>q_{c}$, with baseband instability in the range $0<K^{2}<2 \mu\left|B_{0}\right|^{2} / \delta$. Then, when $L \neq 0$, the instability band in the $K-L$ plane separates from $K=0$ and extends to infinity, see Benney and Roskes (1969). Instability now occurs for all $q$, but as $q$ decreases, the instability band narrows, and is essentially unrealisable for $q<0.5$.

When wind forcing is present $\Delta>0$, and then, $F$ decreases from 1 to 0 , as $s$ increases from 0 to $\infty$ when also $T$ increases from 0 to $\infty$. Although now, the general solution of (21) can be obtained in terms of modified Bessel functions of imaginary order, see Leblanc (2007), qualitatively, it is clear that exponential growth and hence instability will arise when $\left.M F-2 v\left|B_{0}\right|^{2}\right) M<0$, In particular, as $T \rightarrow \infty$. $s \rightarrow \infty, F \rightarrow 0$ and we expect instability for just $v M>0$, independent of $\left|B_{0}\right|$. That is, the dispersive term in the usual modulation instability criterion has been suppressed, and the outcome is that found by Hayes (1973) using Whitham modulation theory for nonlinear waves. In this limit, $s \rightarrow \infty, p \sim F^{1 / 2} \exp \left(\Sigma F^{-1 / 2}\right)$, $\Sigma=|2 M \nu|^{1 / 2} / \delta$. Even taking account of the cancellation of the factor $\tau^{-1 / 2}$ with the pre-factor $\exp (\Delta T)$ in (12), we see that the modulation growth rate is now super-exponential. As noted by Grimshaw (2019), in the deep-water limit $q \rightarrow \infty$, when $\beta \rightarrow 0, v<0$, the criterion $v M>0$ for instability becomes the quarter space $K^{2}>2 L^{2}$. In general, in finite depth, the instability zones are either $\delta K^{2}+\delta_{1} L^{2}<0$ combined with $\mu \alpha K^{2}+(\beta+\mu) L^{2}<0$, or the converse, $\delta K^{2}+\delta_{1} L^{2}>0$ combined with $\mu \alpha K^{2}+(\beta+\mu) L^{2}>0$. In deep water $q>q_{c}=1.363, \mu<0$ and noting that $\beta>0$ it follows that then, $v<0$ for all $K . L$, and then, the instability region is the quarter plane $K^{2}>\delta_{1} L^{2} /|\delta|$. However, in shallow water, $q<q_{c}=1.363, \mu>0$ and it can be shown that $\mu+\beta<0$, and therefore, the instability region has two boundaries $\delta K^{2}+\delta_{1} L^{2}=0$ and $\mu \alpha K^{2}+(\beta+\mu) L^{2}=0$, with instability in the segment between these boundaries, see Benney and Roskes (1969), Hayes (1973). The two boundaries coincide when $q=0.38$, Except for this exceptional value, there is instability for all $q$, but in an increasingly narrow segment as $q$ decreases.

\subsection{Nonlinear development}

In general, the development of a modulation instability into the nonlinear regime requires numerical simulations of the full Benney-Roskes system $(13,14)$ which is not integrable. We shall not attempt numerical simulation here, but some comments and preliminary analysis are presented. In one horizontal space dimension, it is known that modulation instability in the absence of wind forcing can lead to the generation of a Peregrine breather, see, for instance, Kharif et al. (2009), Osborne (2010), Grimshaw and Tovbis (2013). Furthermore, it is conjectured that again, in one horizontal space dimension, the occurrence of rogue waves, represented by breathers, is linked to baseband instability, that is, the domain in modulation wavenumber space, where instability occurs should include the origin $K=0$, see, for instance, Chow et al. (2019). That is satisfied here in deep water when $q>q_{c}$, but fails in shallow water $q<q_{c}$, suggesting that then the occurrence of rogue waves is inhibited. It is not known whether a similar outcome is to be expected when the instability is triggered with a wavenumber $(K, L), L \neq 0$, and there is wind forcing. This is especially the case in one horizontal space dimension, as then there is no modulation instability at all, but even although instability persists in two horizontal space dimensions, the instability domain is a segment in the $K-L$ plane, and contracts to zero at the origin.

Although in general the further exploration of this seems to require numerical simulations, this will not be attempted here. Instead we exploit a reduction to a one-dimensional nonlinear Schrödinger equation, achieved by seeking solutions which depend only on $s, \xi=K X+L Y$, where the wavenumbers $K, L$ are chosen to correspond to a modulation instability. The outcome is a nonlinear Schrödinger equation:

$i B_{s}+M F B_{\xi \xi}+v|B|^{2} B=0$

where $F(s)$ is defined by (15), and $M, v$ are defined by $(20,21)$. Indeed, the modulation instability analysis in 
Sect. 3.1 could be obtained directly from (22). Then, the unforced version of (22) when $F=1$ has a Peregrine breather solution, suggesting that an instability confined to the $\xi$ direction can lead to the formation of a Peregrine breather. If we assume that $\Delta \ll 1$, so that $F(s)$ is slowly varying, then locally, the Peregrine breather solution of (22) is given by, Peregrine (1983)

$$
\begin{aligned}
B_{\mathrm{PB}} & =\alpha\left\{-1+\frac{4(1+2 i v S)}{1+2 \alpha^{2} \nu \xi^{2}(M F)^{-1}+4 v^{2} S^{2}}\right\} \exp (i v S), \\
S & =\int_{0}^{s} \alpha^{2} \mathrm{~d} s .
\end{aligned}
$$

Here, we have assumed that $M v>0$ and $\alpha(s)$ are an amplitude parameter which we allow to vary slowly with $s$. When $F=1, \alpha$ is a constant, and this is the usual Peregrine breather. Here, it evolves slowly, as $s$ increases and $F$ decreases. The determination of how $\alpha(s)$ varies requires sophisticated asymptotic analysis. Instead, an indication of the likely outcome is to make judicious use of the energy conservation law (16), which indicates from simple scaling arguments that $\alpha^{2}\left(F^{1 / 2} / \alpha\right)$ is a constant. Thus, we find that $\alpha F^{1 / 2}=\alpha_{0}$, where $\alpha_{0}$ is the constant amplitude at $s=0, F=1$. Note that then $S=\alpha_{0}^{2}\left(s+\Delta s^{2}\right)$ and that the spatial width in the $\xi$-direction does not change. Hence, as $s$ increases, the breather amplitude increases as $F^{-1 / 2}$ or as $\exp (\Delta T)$. After taking account of the transformation form $A$ to $B$ in (12), this is super-exponential growth, and is exactly twice that for a linearised instability. A similar outcome was found for the envelope soliton solution of (22) by Grimshaw (2019).

\section{Discussion}

The main outcomes of this analysis are that wind forcing enhances modulation instability, which becomes superexponential, and that the domain of instability in the modulation wavenumber space is enlarged. The super-exponential behaviour has been previously noted for deep-water waves in one space dimension, see Leblanc (2007), Touboul et al. (2008), Montalvo et al. (2013), Brunetti et al. (2014), Slunyaev et al. (2015), Grimshaw (2018), and the extension here is to be expected. The enlargement of the domain in the modulation wavenumber space was noted by Grimshaw (2019) for deep-water waves, and again, the extension here to finite depth is to be expected. In addition, significantly, in two horizontal space dimensions, the domain of modulation instability occurs for all $q=k h$ and there is no cutoff at $q=q_{c}=1.363$, although for $q<q_{c}$, the domain of instability shrinks and becomes very narrow for $q<0.5$ making the occurrence of modulation instability unlikely.

The outcome of modulation instability is expected to be the generation of rogue waves, which can be represented within the framework of the nonlinear Schrdinger equation by Peregrine breathers. Hence, we examined here the effect of wind forcing on a Peregrine breather and find that the amplitude increases exponentially at twice the rate of a linear instability. However, we caution that this result is obtained in a one-dimensional horizontal space setting and further studies are needed to examine this in a two-dimensional horizontal space setting. This would seem to require numerical simulations of the forced Benney-Roskes system $(7,8)$, or $(13,14)$, or indeed the Zakharov equations, or the full Euler equations, all well beyond the scope of this paper. However, we note that the prediction of the present theory that the regime for modulation instability is extended in the two-dimensional $K-L$ plane is consistent with the two-dimensional nonlinear wave interactions which form the basis of the Zakharov equations, and are seen in laboratory and field experiments, see the recent reviews by Shemer (2018), Zakharov (2018), for instance.

Acknowledgements This research was supported by a Leverhulme Emeritus Fellowship, EM-2019-030/9.

Open Access This article is distributed under the terms of the Creative Commons Attribution 4.0 International License (http://creativecomm ons.org/licenses/by/4.0/), which permits unrestricted use, distribution, and reproduction in any medium, provided you give appropriate credit to the original author(s) and the source, provide a link to the Creative Commons license, and indicate if changes were made.

\section{References}

Benney DJ, Roskes GJ (1969) Wave instabilities. Stud Appl Math 48:377-385

Brunetti M, Marchiando N, Berti N, Kasparian J (2014) Nonlinear fast growth of water waves under wind forcing. Phys Lett A 378:10251030

Chow KW, Chan HN, Grimshaw RH (2019) Modulation instability of internal waves in a smoothly stratified shallow fluid with a constant buoyancy frequency. Nat Hazards Earth Syst Sci Br Commun 19:583-587

Davey A, Stewartson K (1974) On three-dimensional packets of surface waves. Proc R Soc A 338:101-110

Djordjevic VD, Redekopp LG (1977) On two dimensional packets of capillary-gravity waves. J Fluid Mech 79:703-714

Grimshaw R (2007) Envelope solitary waves. In: Grimshaw R (ed) Solitary waves in fluids: advances in fluid mechanics, vol 45. WIT Press, Southampton, pp 159-179

Grimshaw R (2018) Generation of wave groups. In: Grimshaw R, Hunt J, Johnson E (eds) IUTAM symposium wind waves, vol 26. Elsevier IUTAM Procedia Series, Amsterdam, pp 99-101

Grimshaw R (2019) Generation of wave groups by shear layer instability. Fluids 4:39

Grimshaw R, Tovbis A (2013) Rogue waves: analytical predictions. Proc R Soc 469:20130094

Hasimoto H, Ono H (1972) Nonlinear modulation of gravity waves. J Phys Soc Jpn 33:805-811

Hayes WD (1973) Group velocity and nonlinear dispersive wave propagation. Proc R Soc A 333:199-221 
Janssen P (2004) The interaction of ocean waves and wind. Cambridge University Press, Cambridge

Kharif C, Pelinovsky E, Slunyaev A (2009) Rogue waves in the ocean. In: Advances in geophysical and environmental mechanics and mathematics, vol 14. Springer, New York

Leblanc S (2007) Amplification of nonlinear surface waves by wind. Phys Fluids 19:101705

Miles JW (1957) On the generation of surface waves by shear flows. J Fluid Mech 3:185-204

Miles JW (1993) Surface wave generation revisited. J Fluid Mech 256:427-441

Montalvo P, Kraenkel R, Manna MA, Kharif C (2013) Wind-wave amplification mechanisms: possible models for steep wave events in finite depth. Nat Hazards Earth Syst Sci 13:2805-2813

Morland LC, Saffman PG (1993) Effect of wind profile on the instability of wind blowing over water. J Fluid Mech 252:383-398

Osborne AR (2010) Nonlinear ocean waves and the inverse scattering transform. Elseveier, Amsterdam

Peregrine DH (1983) Water waves, nonlinear Schrödinger equations, and their solutions. J Aust Math Soc Ser B 25(1):16-43

Sajjadi SG, Hunt JCR, Drullion F (2014) Asymptotic multi-layer analysis of wind over unsteady monochromatic surface waves. J Eng Math 84:73-85
Shemer L (2018) Laboratory study of temporal and spatial evolution of waves excited on water surface initially at rest by impulsive wind forcing. In: Grimshaw R, Hunt J, Johnson E (eds) IUTAM symposium wind waves, vol 26. Elsevier IUTAM Procedia Series, pp 153-161

Slunyaev A, Sergeeva A, Pelinovsky E (2015) Wave amplification in the framework of forced nonlinear schrödinger equation: the rogue wave context. Physica D 301:18-27

Stiassnie M, Agnon Y, Janssen PAEM (2007) Temporal and spatial growth of wind waves. J Phys Ocean 37:106-114

Touboul J, Kharif C, Pelinovsky E, Giovanangeli J-P (2008) On the interaction of wind and steep gravity wave groups using Miles' and Jeffreys' mechanisms. Nonlinear Proc Geophys 15:1023-1031

Zakharov V (2018) Analytic theory of a wind-driven sea. In: Grimshaw R, Hunt J, Johnson E (eds) IUTAM symposium wind waves, vol 26. Elsevier IUTAM Procedia Series, Amsterdam, pp 43-58

Zakharov VE, Resio D, Pushkarev A (2017) Balanced source terms for wave generation within the Hasselmann equation. Nonlinear Proc Geophys 24:581-597

Publisher's Note Springer Nature remains neutral with regard to jurisdictional claims in published maps and institutional affiliations. 